\title{
Assessment of New Expanded Porpholactones as UV/Vis/NIR Chromophores for Dye- Sensitized Solar Cell Applications
}

\author{
Ricardo Pino-Rios ${ }^{\text {a*; }}$ Nicolás Montenegro-Pohlhammer ${ }^{\text {a.b }}$; Gloria Cárdenas-Jirón ${ }^{\mathrm{a}}$

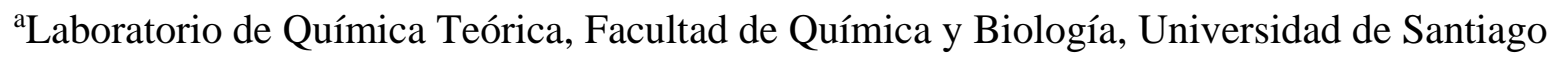 \\ de Chile (USACH), Santiago, Chile. ricardo.pino@usach.cl \\ bDepartamento de Química Física. Universidad de Sevilla. c/ Profesor García González, \\ s/n. 41012 Sevilla. Spain.
}

\section{SUPPORTING INFORMATION}

Figure S1. Decomposed vibrational resolved absorption spectrum for Sapphyrin.

Figure S2. Decomposed vibrational resolved absorption spectrum for all M1.

Figure S3. Decomposed vibrational resolved absorption spectrum for all M2.

Figure S4. Decomposed vibrational resolved absorption spectrum for all C1.

Figure S5. Decomposed vibrational resolved absorption spectrum for all C2.

Figure S6. Decomposed vibrational resolved absorption spectrum for all $\mathbf{T}$.

Figure S7. Ionic resonant structures of compounds M1, C1 and $\mathbf{T}$ compounds.

Table S1. Molecular Orbital contributions of the characteristic bands of sapphyrins, expanded porpholactones and porphodilactones.

Figure S8. Transmission Spectrum and Projected Density of States of Sapphyrin.

Figure S9. Transmission Spectrum and Projected Density of States of M1.

Figure S10. Transmission Spectrum and Projected Density of States of M2.

Figure S11. Transmission Spectrum and Projected Density of States of C1.

Figure S12. Transmission Spectrum and Projected Density of States of C2.

Figure S13. Transmission Spectrum and Projected Density of States of T. 
Figure S1. Decomposed vibrational resolved absorption spectrum for sapphyrin at the M06-2x/6-311G** level of theory.

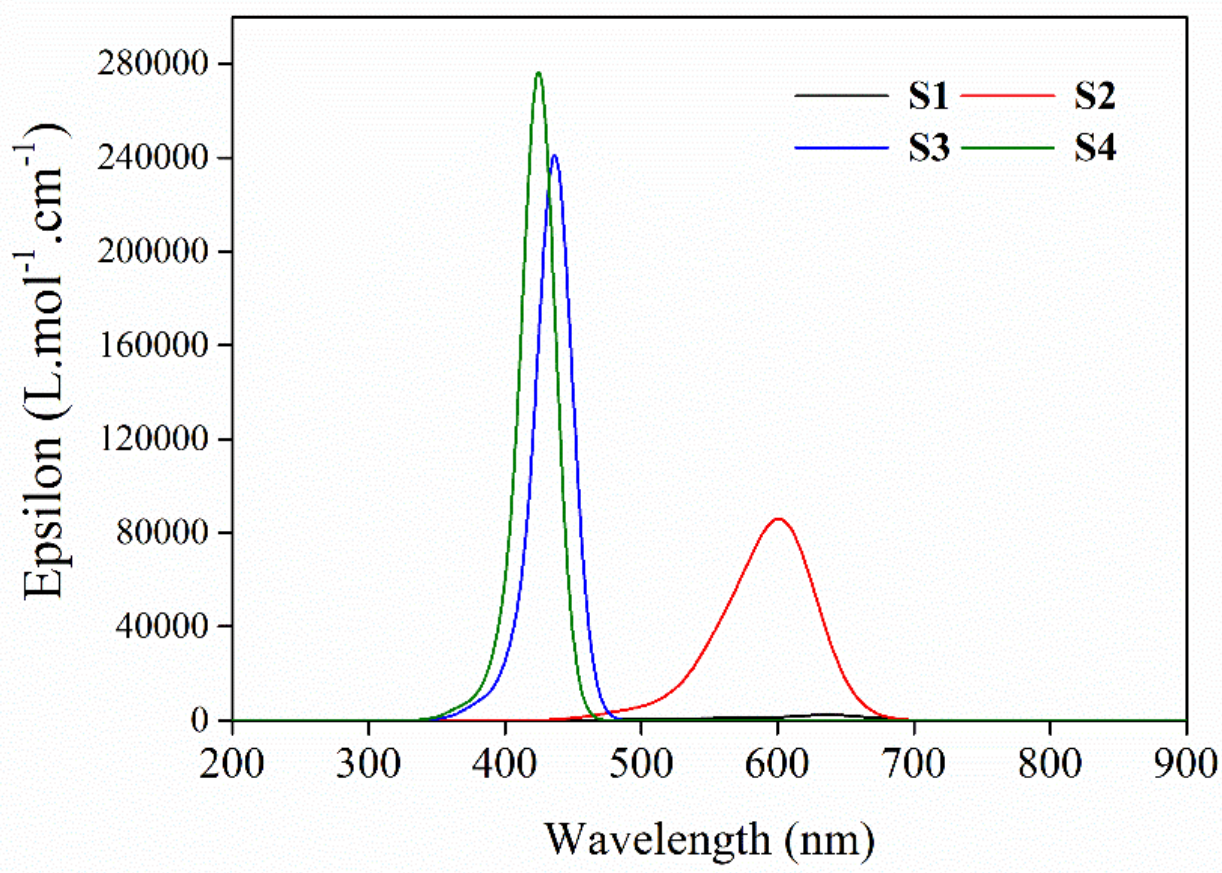


Figure S2. Decomposed vibrational resolved absorption spectrum for all M1 at the M06$2 \mathrm{x} / 6-311 \mathrm{G}^{* *}$ level of theory.

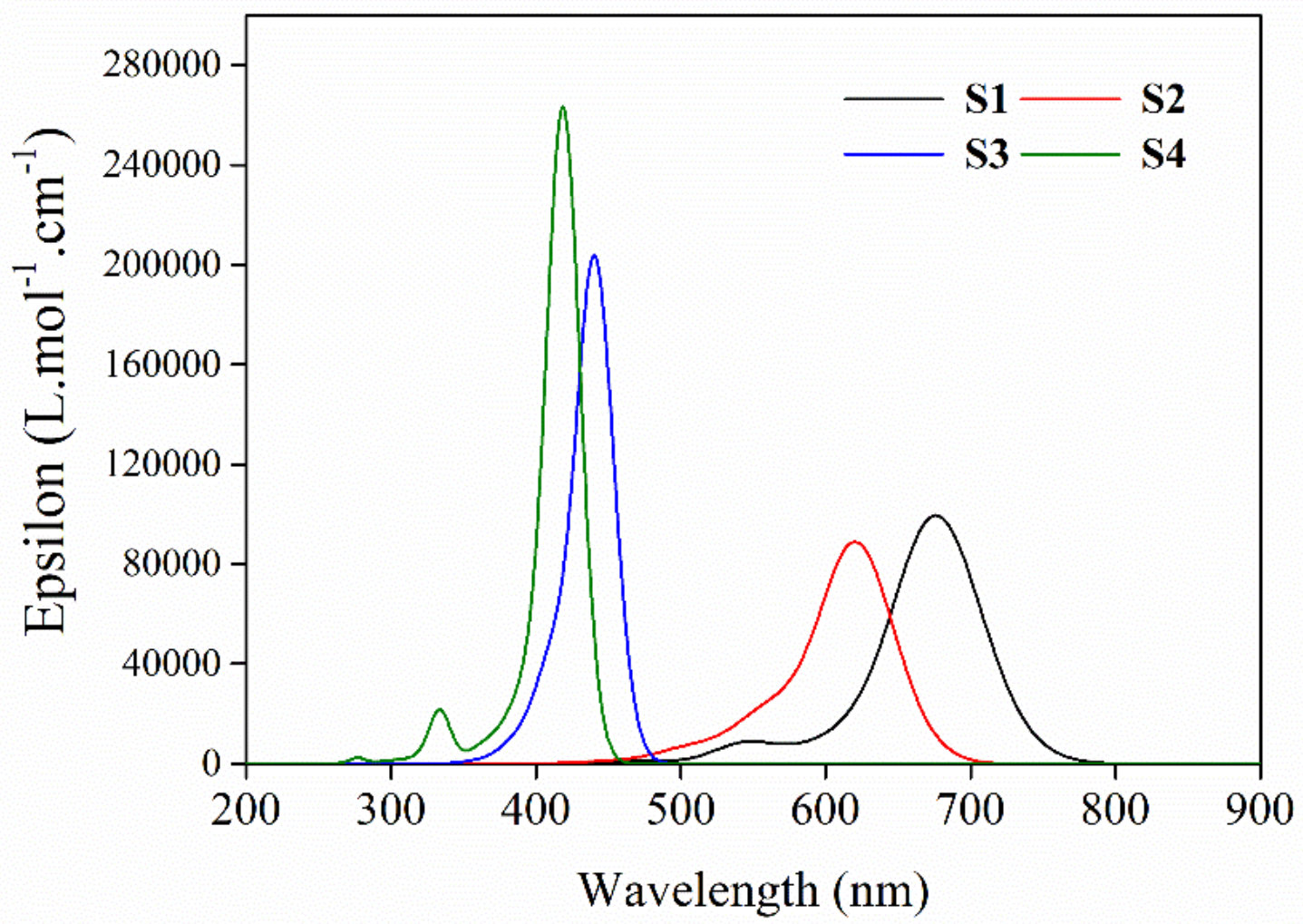


Figure S3. Decomposed vibrational resolved absorption spectrum for all M2 at the M06$2 \mathrm{x} / 6-311 \mathrm{G}^{* *}$ level of theory.

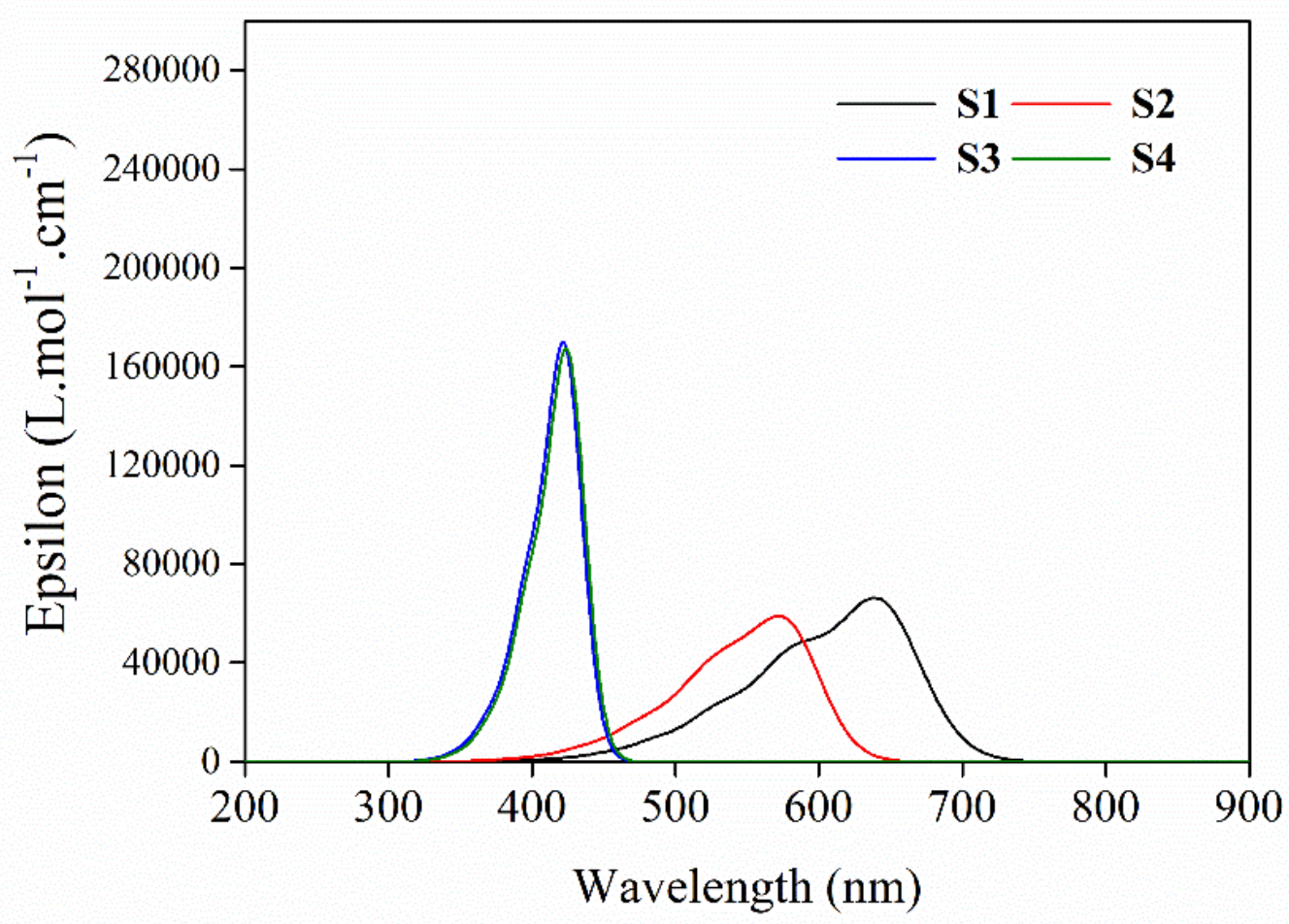


Figure S4. Decomposed vibrational resolved absorption spectrum for all $\mathbf{C 1}$ at the M06$2 \mathrm{x} / 6-311 \mathrm{G}^{* *}$ level of theory.

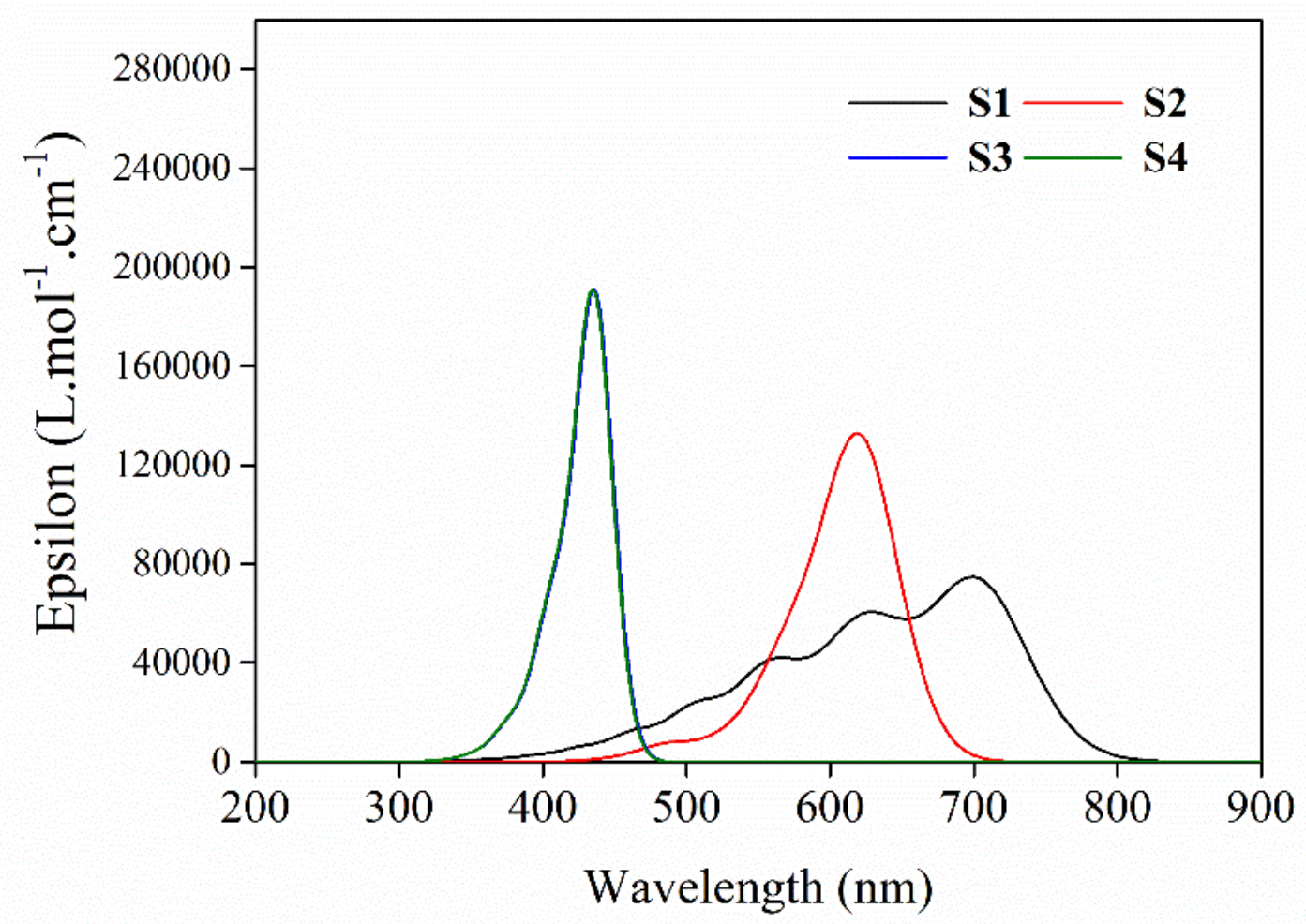


Figure S5. Decomposed vibrational resolved absorption spectrum for all $\mathbf{C 2}$ at the M06$2 \mathrm{x} / 6-311 \mathrm{G}^{* *}$ level of theory.

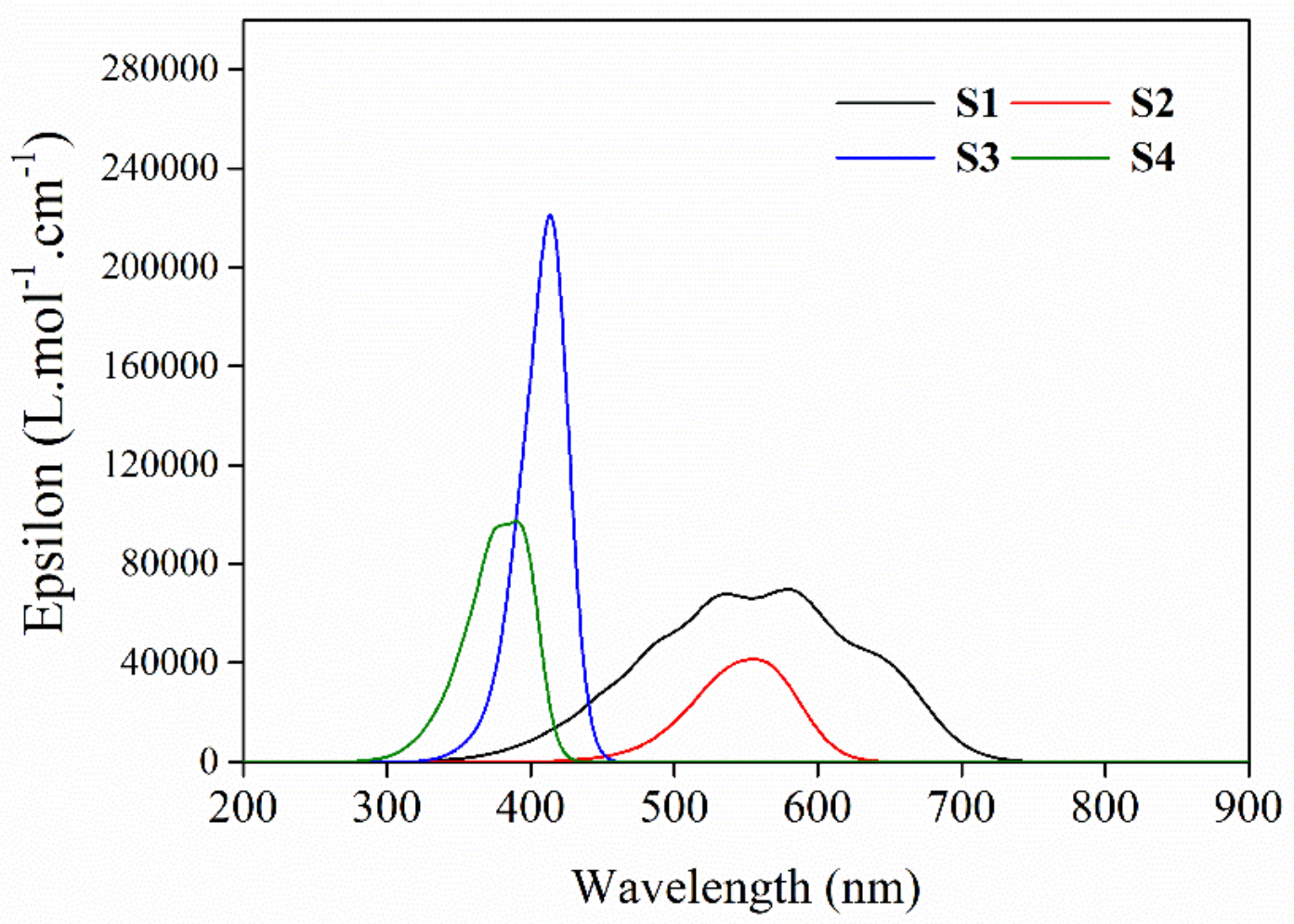


Figure S6. Decomposed vibrational resolved absorption spectrum for all $\mathbf{T}$ at the M062X/6-311G** level of theory.

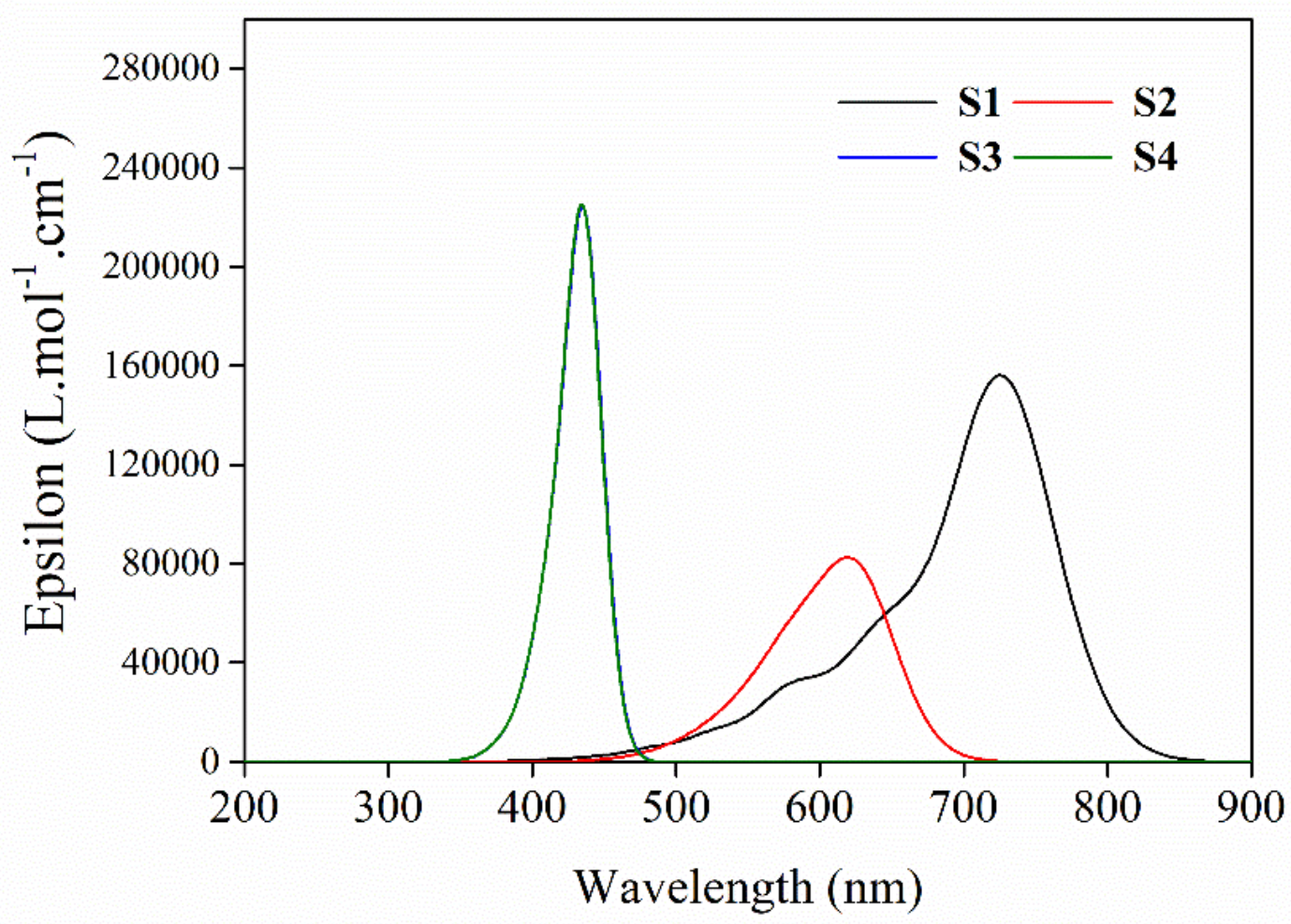


Figure S7. Ionic resonant structures of compounds M1, C1 and T compounds.

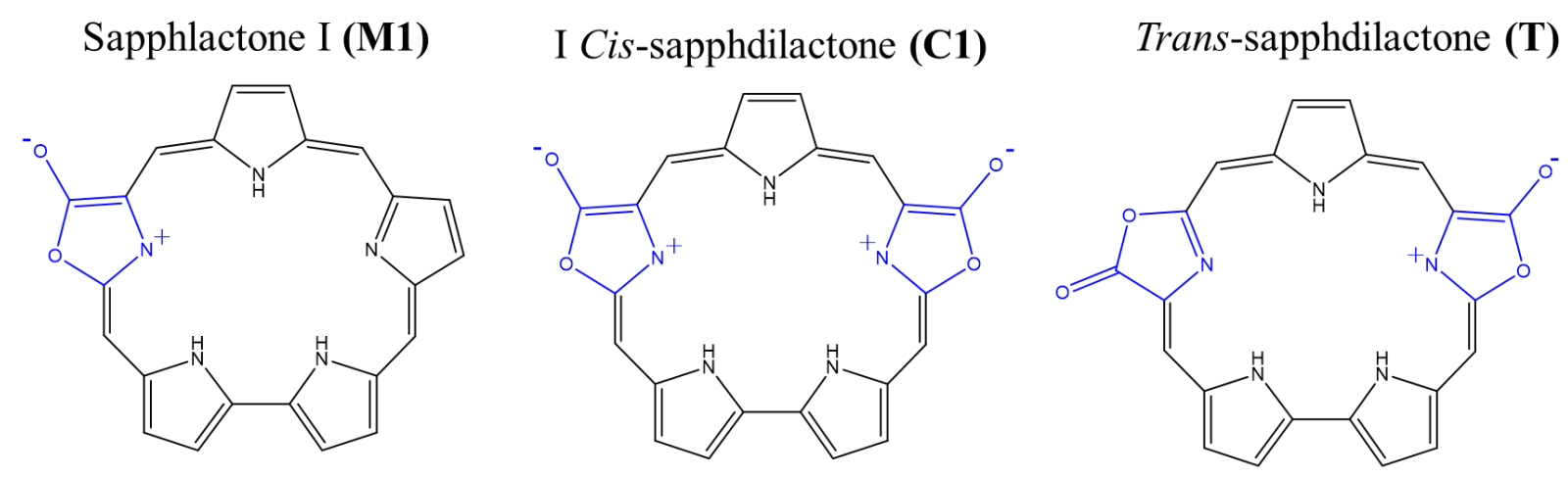


Table S1. Molecular orbital contributions of the characteristic bands of sapphyrins, expanded porpholactones and porphodilactones at the M06-2X/6-311G** leve lof theory.

\begin{tabular}{|c|c|c|c|}
\hline System & Band & Orbital Composition & \% Contribution \\
\hline \multirow{8}{*}{ Sapph } & \multirow{2}{*}{ Qx } & H-1 $\rightarrow \mathbf{L}$ & 51.5 \\
\hline & & $\mathrm{H} \rightarrow \mathrm{L}+\mathbf{1}$ & 49.0 \\
\hline & \multirow{2}{*}{ Qy } & H-1 $\rightarrow$ L+1 & 21.5 \\
\hline & & $\mathbf{H} \rightarrow \mathbf{L}$ & 78.8 \\
\hline & \multirow{2}{*}{$\mathbf{B x}$} & H-1 $\rightarrow \mathbf{L}$ & 49.0 \\
\hline & & $\mathrm{H} \rightarrow \mathrm{L}+\mathbf{1}$ & 52.8 \\
\hline & \multirow{2}{*}{ By } & H-1 $\rightarrow$ L+1 & 60.7 \\
\hline & & $\mathbf{H} \rightarrow \mathbf{L}$ & 40.9 \\
\hline \multirow{16}{*}{ M1 } & \multirow{4}{*}{ Qx } & $\mathbf{H}-\mathbf{1} \rightarrow \mathbf{L}$ & 26.5 \\
\hline & & H-1 $\rightarrow$ L+1 & 11.9 \\
\hline & & $\mathbf{H} \rightarrow \mathbf{L}$ & 51.1 \\
\hline & & $\mathbf{H} \rightarrow \mathbf{L}+\mathbf{1}$ & 11.1 \\
\hline & \multirow{4}{*}{ Qy } & H-1 $\rightarrow$ L & 44.0 \\
\hline & & $\mathrm{H}-1 \rightarrow \mathrm{L}+\mathbf{1}$ & 6.2 \\
\hline & & $\mathbf{H} \rightarrow \mathbf{L}$ & 30.3 \\
\hline & & $\mathrm{H} \rightarrow \mathrm{L}+\mathbf{1}$ & 20.0 \\
\hline & \multirow{4}{*}{$\mathbf{B x}$} & H-1 $\rightarrow$ L & 25.9 \\
\hline & & H-1 $\rightarrow$ L+1 & 21.3 \\
\hline & & $\mathbf{H} \rightarrow \mathbf{L}$ & 7.6 \\
\hline & & $\mathrm{H} \rightarrow \mathrm{L}+\mathbf{1}$ & 47.5 \\
\hline & \multirow{4}{*}{ By } & H-1 $\rightarrow \mathbf{L}$ & 29.5 \\
\hline & & H-1 $\rightarrow$ L+1 & 18.0 \\
\hline & & $\mathbf{H} \rightarrow \mathbf{L}$ & 7.6 \\
\hline & & $\mathrm{H} \rightarrow \mathrm{L}+\mathbf{1}$ & 47.2 \\
\hline \multirow{14}{*}{ M2 } & \multirow{3}{*}{ Qx } & H-1 $\rightarrow \mathbf{L}$ & 4.3 \\
\hline & & H-1 $\rightarrow$ L+1 & 19.7 \\
\hline & & $\mathbf{H} \rightarrow \mathbf{L}$ & 74.7 \\
\hline & \multirow{3}{*}{ Qy } & H-1 $\rightarrow \mathbf{L}$ & 5.6 \\
\hline & & H-1 $\rightarrow$ L+1 & 17.8 \\
\hline & & $\mathbf{H} \rightarrow \mathbf{L}$ & 75.0 \\
\hline & \multirow{4}{*}{$\mathbf{B x}$} & H-1 $\rightarrow \mathbf{L}$ & 22.7 \\
\hline & & H-1 $\rightarrow$ L+1 & 28.2 \\
\hline & & $\mathbf{H}+\mathbf{L}$ & 11.0 \\
\hline & & $\mathrm{H}+\mathrm{L}+\mathbf{1}$ & 39.5 \\
\hline & \multirow{4}{*}{ By } & H-1 $\rightarrow$ L & 22.6 \\
\hline & & H-1 $\rightarrow$ L+1 & 28.3 \\
\hline & & $\mathbf{H}+\mathbf{L}$ & 11.0 \\
\hline & & $\mathrm{H}+\mathrm{L}+1$ & 39.5 \\
\hline
\end{tabular}




\begin{tabular}{|c|c|c|c|}
\hline \multirow{10}{*}{ C1 } & \multirow{2}{*}{ Qx } & $\mathrm{H}-1 \rightarrow \mathrm{L}+1$ & 13.4 \\
\hline & & $\mathbf{H} \rightarrow \mathbf{L}$ & 87.4 \\
\hline & \multirow{2}{*}{ Qy } & $\mathrm{H}-1 \rightarrow \mathrm{L}+1$ & 15.3 \\
\hline & & $\mathbf{H} \rightarrow \mathbf{L}$ & 84.9 \\
\hline & \multirow{3}{*}{$\mathbf{B x}$} & $\mathbf{H}-1 \rightarrow \mathbf{L}$ & 32.9 \\
\hline & & H-1 $\rightarrow$ L+1 & 3.8 \\
\hline & & $\mathrm{H} \rightarrow \mathrm{L}+\mathbf{1}$ & 65.1 \\
\hline & \multirow{3}{*}{ By } & H-1 $\rightarrow$ L & 32.9 \\
\hline & & H-1 $\rightarrow$ L+1 & 3.7 \\
\hline & & $\mathrm{H} \rightarrow \mathrm{L}+\mathbf{1}$ & 65.2 \\
\hline \multirow{8}{*}{$\mathrm{C} 2$} & \multirow{2}{*}{ Qx } & $\mathrm{H}-1 \rightarrow \mathrm{L}+1$ & 12.0 \\
\hline & & $\mathbf{H} \rightarrow \mathbf{L}$ & 88.8 \\
\hline & \multirow{2}{*}{ Qy } & H-1 $\rightarrow \mathbf{L}$ & 75.3 \\
\hline & & $H \rightarrow L+1$ & 24.4 \\
\hline & \multirow{2}{*}{$\mathbf{B x}$} & $\mathbf{H}-1 \rightarrow \mathrm{L}$ & 43.9 \\
\hline & & $\mathrm{H} \rightarrow \mathrm{L}+\mathbf{1}$ & 57.2 \\
\hline & \multirow{2}{*}{ By } & H-1 $\rightarrow$ L+1 & 83.3 \\
\hline & & $\mathbf{H} \rightarrow \mathbf{L}$ & 18.1 \\
\hline \multirow{12}{*}{$\mathbf{T}$} & \multirow{3}{*}{ Qx } & $\mathbf{H}-1 \rightarrow \mathbf{L}$ & 3.1 \\
\hline & & $\mathrm{H}-1 \rightarrow \mathrm{L}+1$ & 9.9 \\
\hline & & $\mathbf{H} \rightarrow \mathbf{L}$ & 86.9 \\
\hline & \multirow{3}{*}{ Qy } & $\mathbf{H}-1 \rightarrow \mathbf{L}$ & 63.8 \\
\hline & & $\mathbf{H} \rightarrow \mathbf{L}$ & 18.3 \\
\hline & & $\mathbf{H} \rightarrow \mathbf{L}+\mathbf{1}$ & 16.5 \\
\hline & \multirow{3}{*}{$\mathbf{B x}$} & H-1 $\rightarrow \mathbf{L}$ & 34.3 \\
\hline & & $\mathrm{H}-1 \rightarrow \mathrm{L}+1$ & 4.4 \\
\hline & & $\mathrm{H} \rightarrow \mathrm{L}+\mathbf{1}$ & 63.3 \\
\hline & \multirow{3}{*}{ By } & $\mathbf{H}-1 \rightarrow \mathbf{L}$ & 34.1 \\
\hline & & $\mathrm{H}-1 \rightarrow \mathrm{L}+1$ & 4.9 \\
\hline & & $\mathbf{H} \rightarrow \mathbf{L}+\mathbf{1}$ & 62.8 \\
\hline
\end{tabular}


Figure S8. Transmission Spectrum and Projected Density of States of Sapphyrin

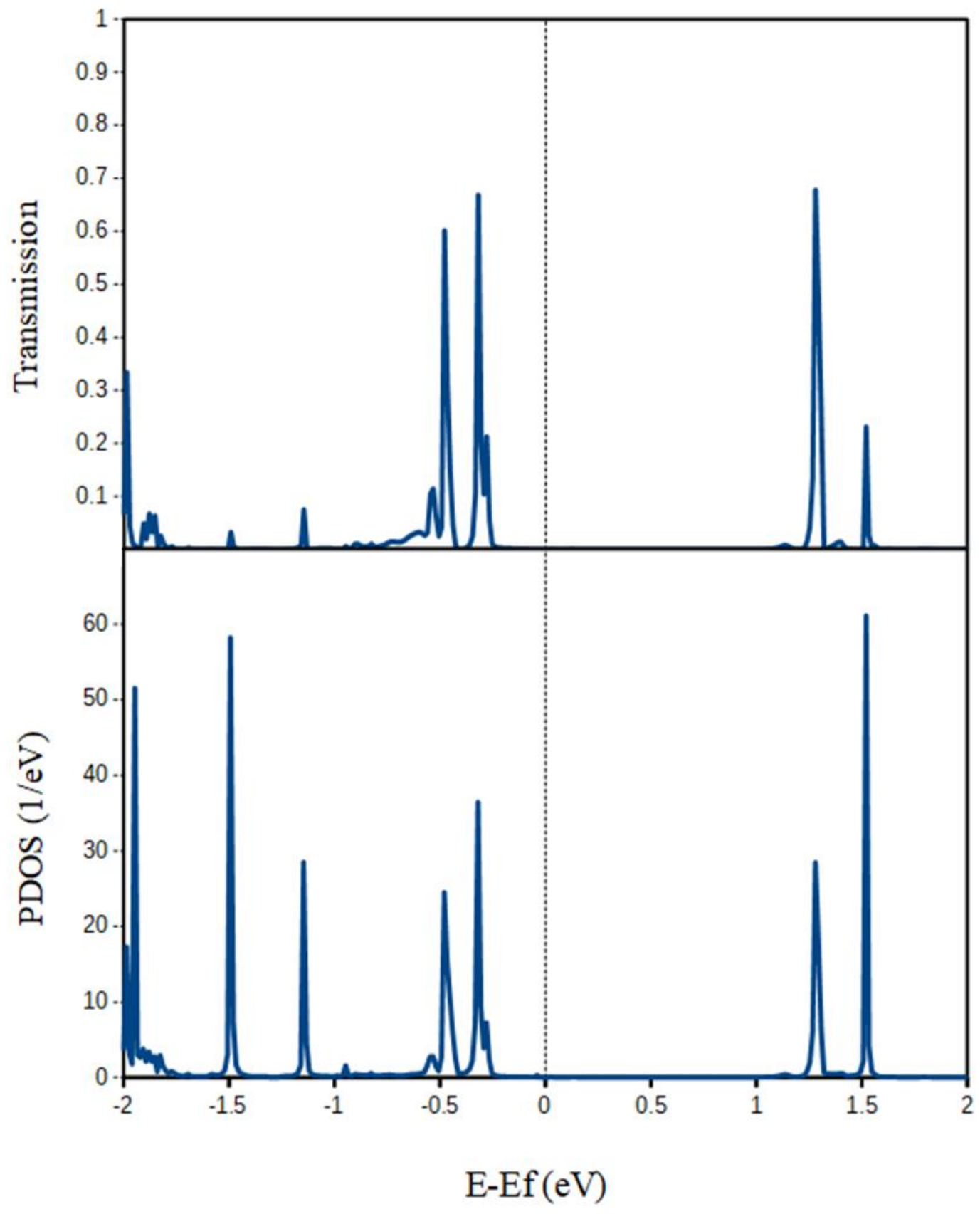


Figure S9. Transmission Spectrum and Projected Density of States of M1.

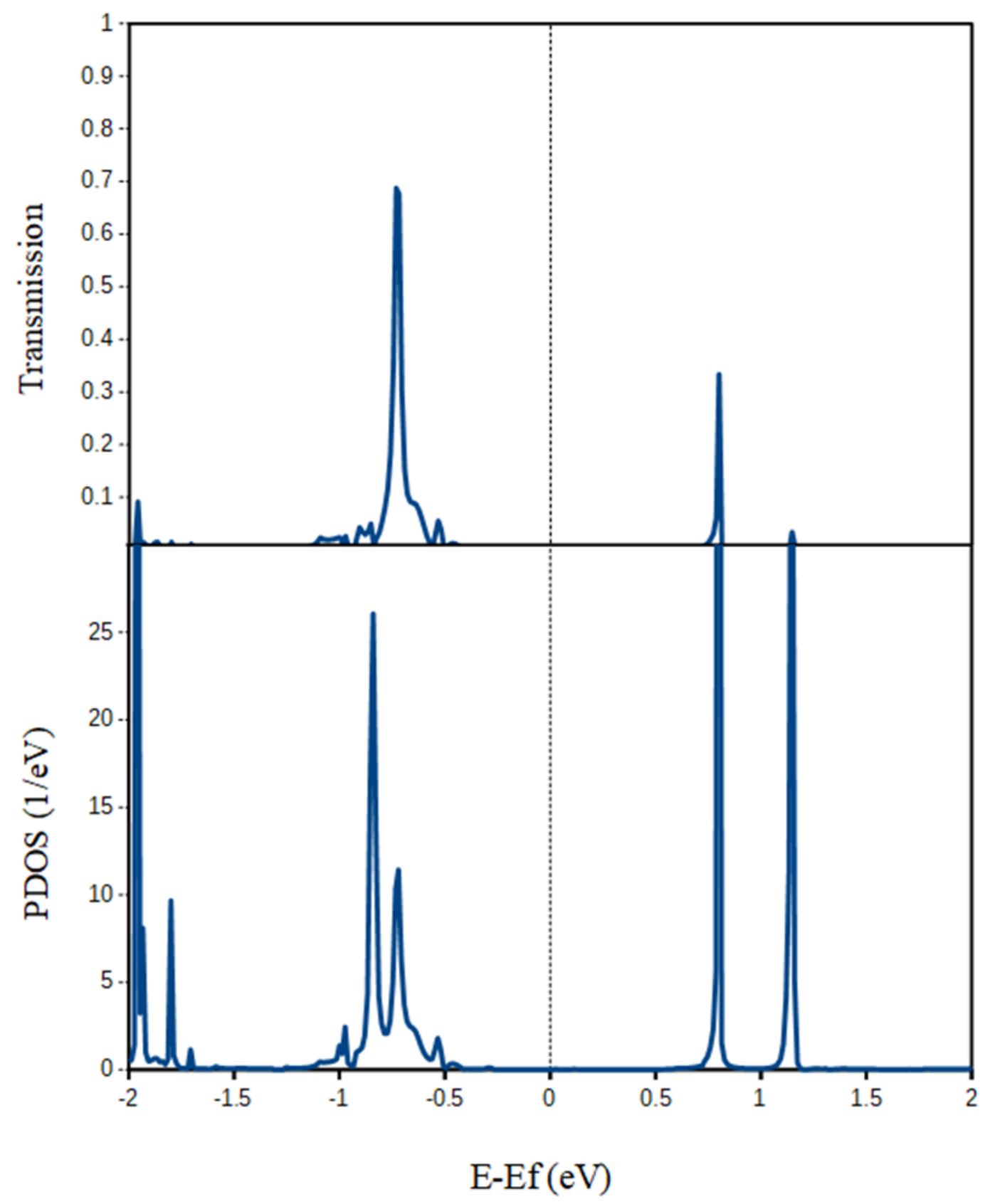


Figure S10. Transmission Spectrum and Projected Density of States of M2.

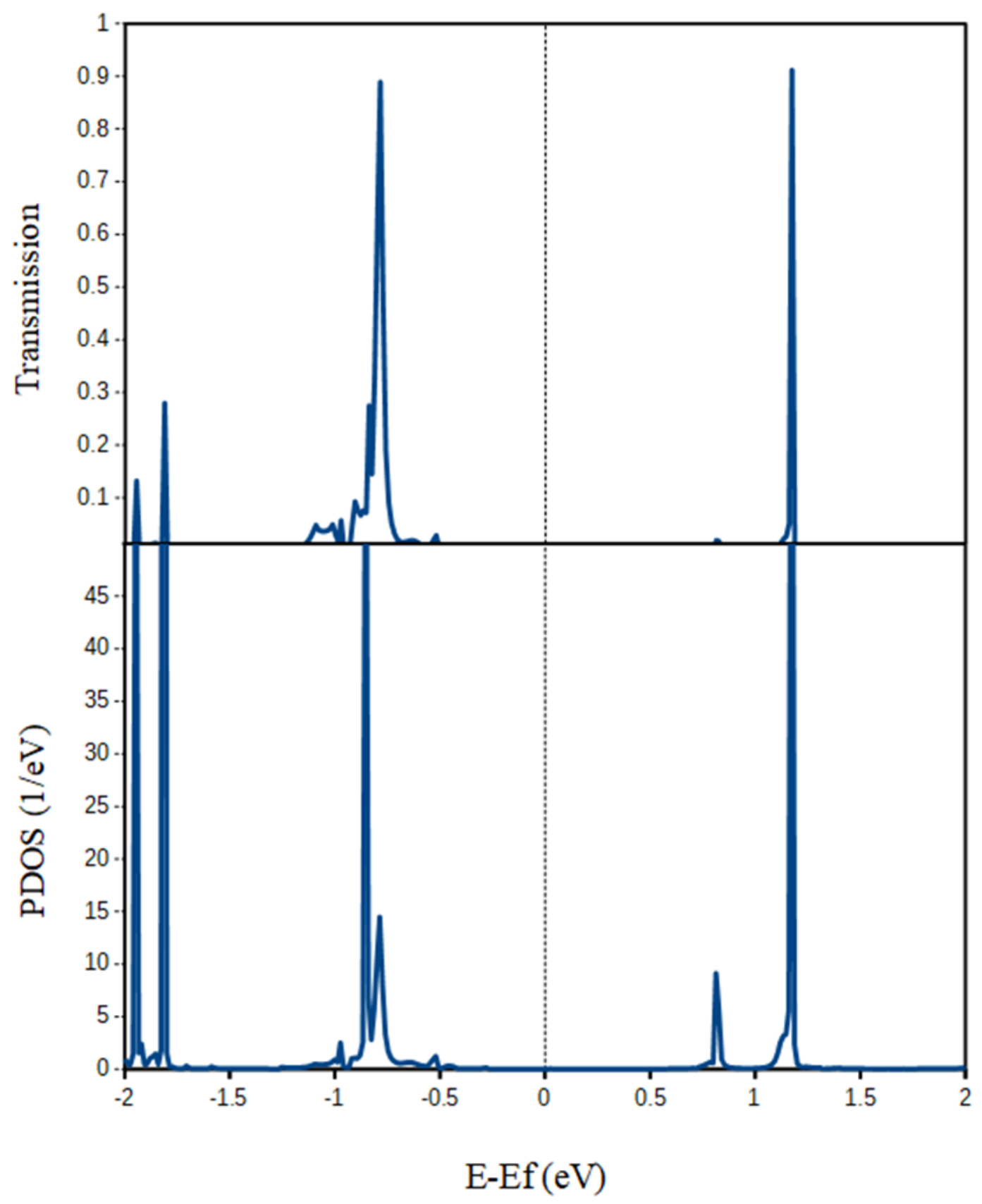


Figure S11. Transmission Spectrum and Projected Density of States of C1.

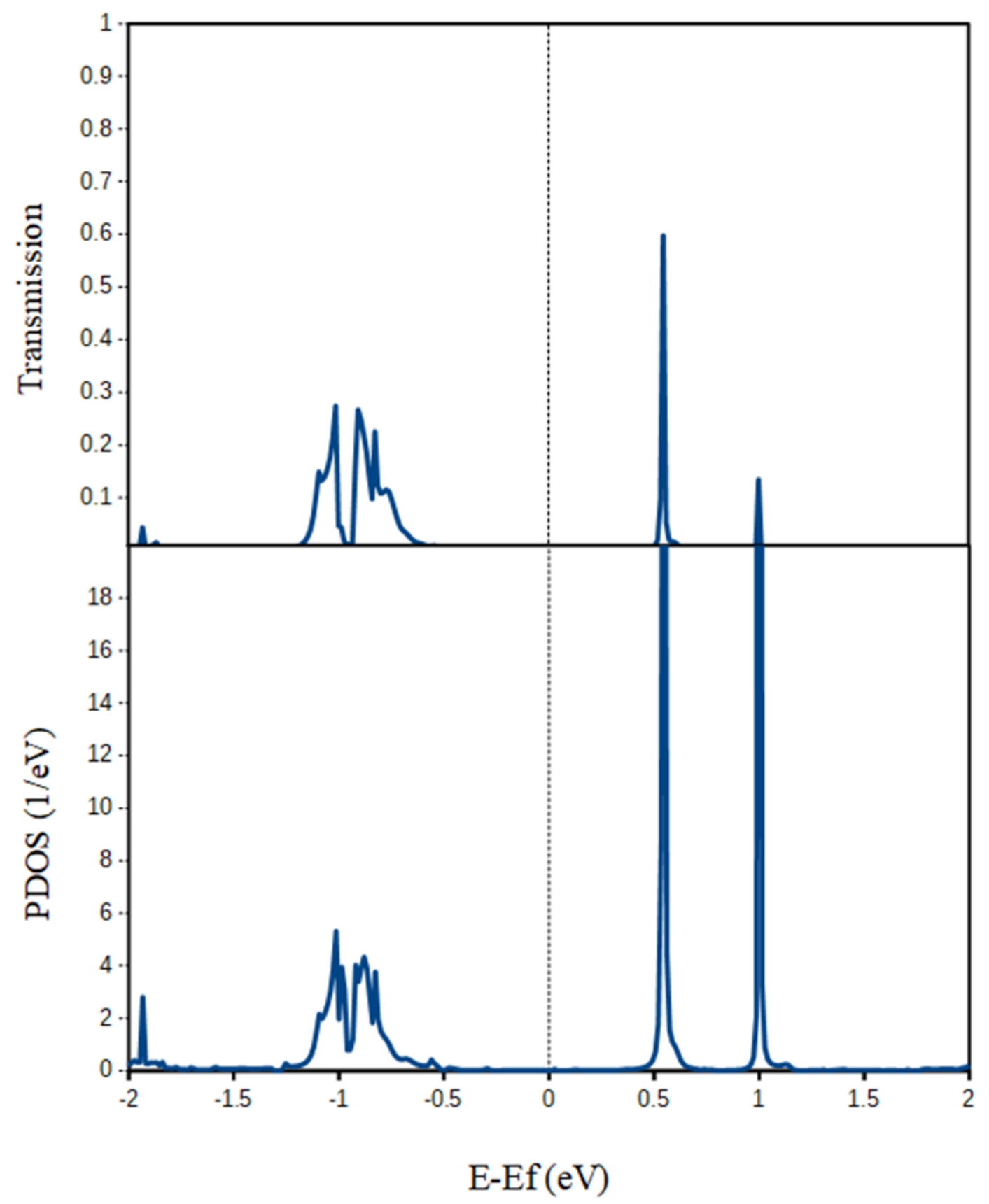


Figure S12. Transmission Spectrum and Projected Density of States of C2.

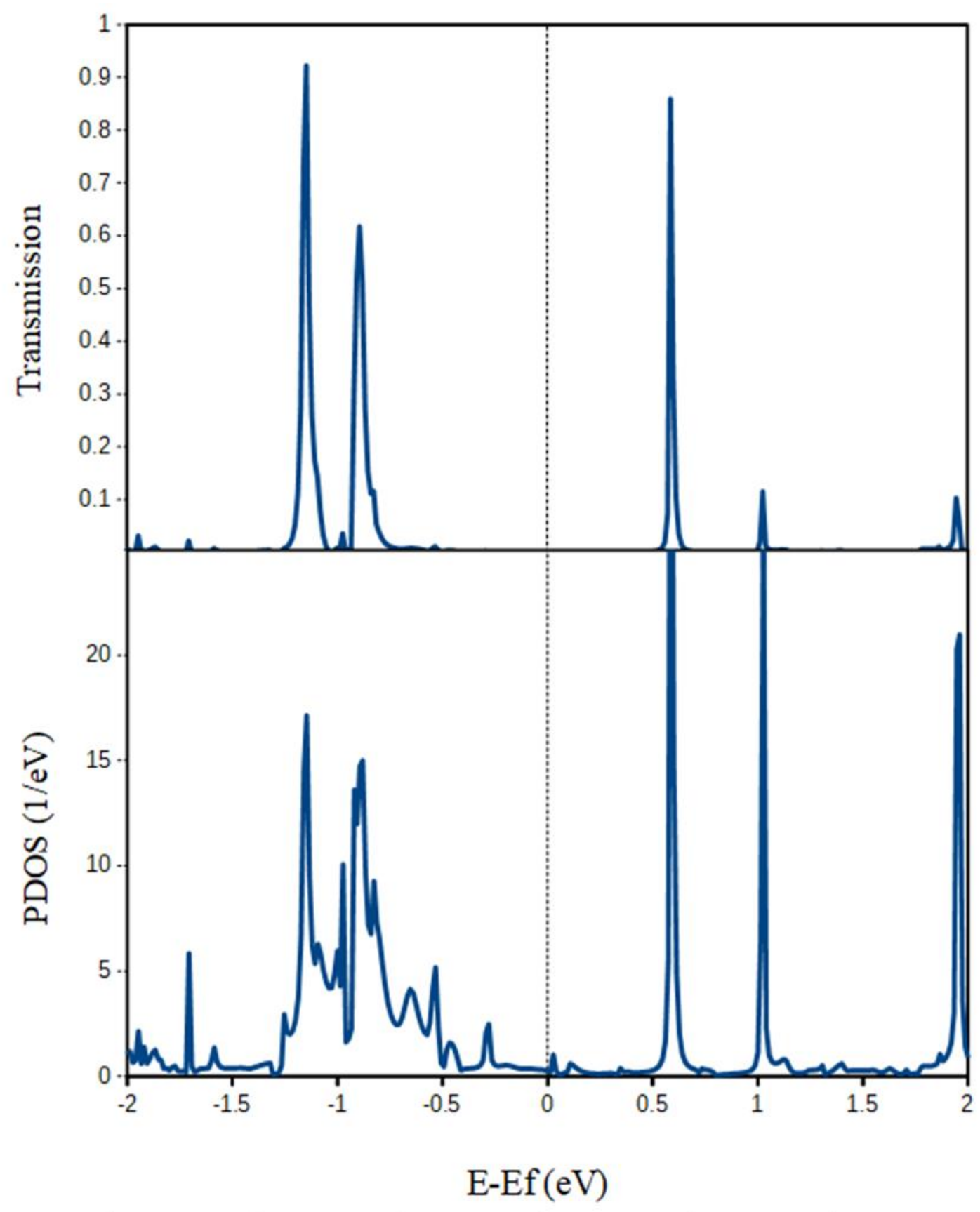


Figure S13. Transmission Spectrum and Projected Density of States of T.

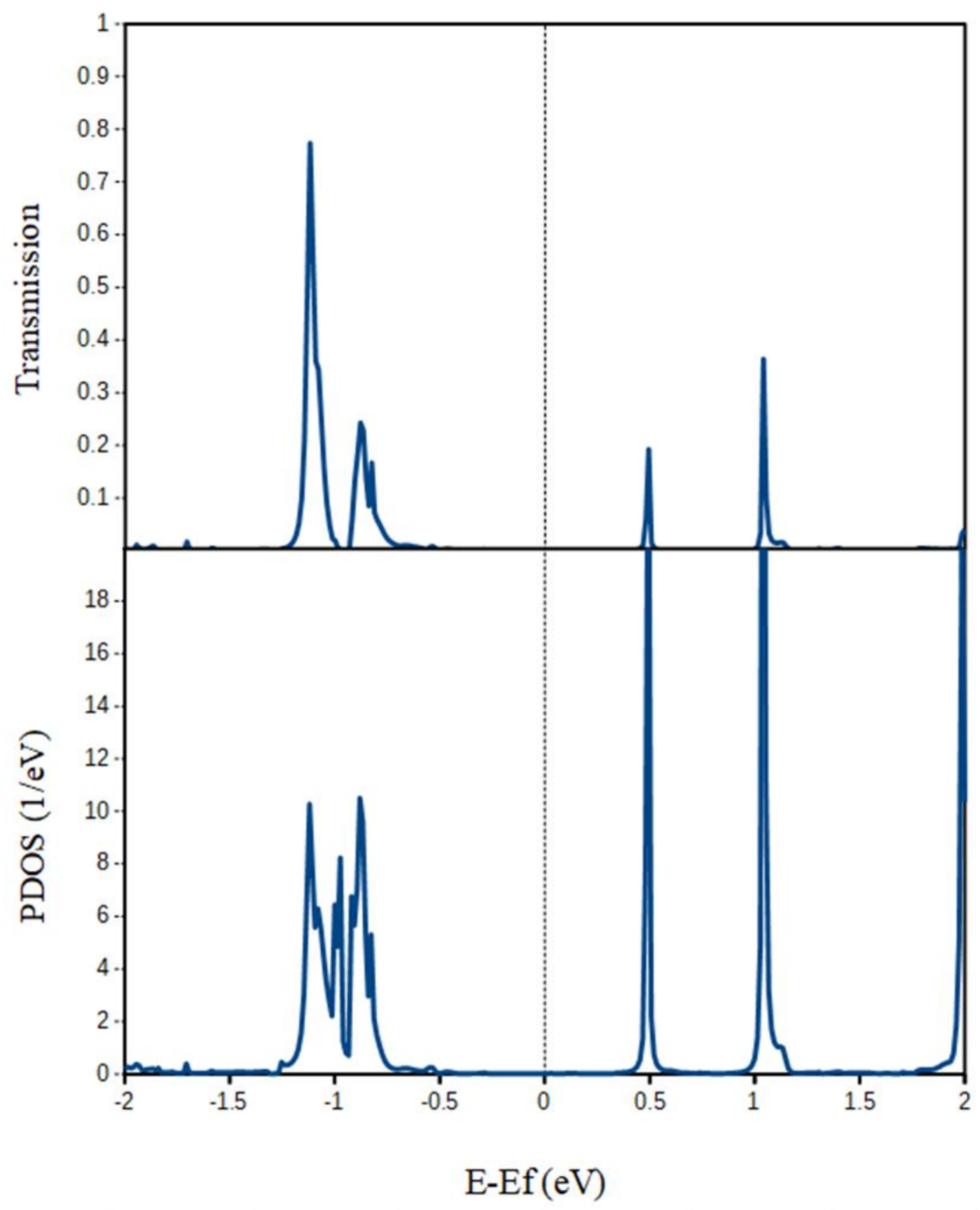


Table S2. Cartesian coordinates for studied compounds at the M06-2x/6-311G** level of theory.

\begin{tabular}{|c|c|c|c|c|c|c|c|}
\hline \multicolumn{4}{|c|}{ Sapphyrin } & \multicolumn{4}{|c|}{ M1 } \\
\hline 6 & 2.848880000 & -3.856138000 & -0.039049000 & 6 & 1.493814000 & 4.523610000 & 0.000028000 \\
\hline 6 & 1.539072000 & -4.291251000 & -0.031348000 & 6 & 0.119431000 & 4.600921000 & 0.000008000 \\
\hline 6 & 0.708739000 & -3.149519000 & -0.007671000 & 6 & -0.388971000 & 3.280129000 & 0.000000000 \\
\hline 6 & 2.833855000 & -2.445872000 & -0.020519000 & 6 & 1.851247000 & 3.152102000 & 0.000009000 \\
\hline 7 & 1.517790000 & -2.053328000 & -0.003239000 & 7 & 0.689726000 & 2.433229000 & -0.000032000 \\
\hline 6 & -0.708738000 & -3.149519000 & 0.007671000 & 6 & -1.736936000 & 2.893968000 & -0.000002000 \\
\hline 6 & -1.539072000 & -4.291251000 & 0.031348000 & 6 & -2.861558000 & 3.770340000 & 0.000010000 \\
\hline 6 & -2.848879000 & -3.856139000 & 0.039048000 & 6 & -3.991605000 & 2.999361000 & 0.000009000 \\
\hline 6 & -2.833855000 & -2.445872000 & 0.020519000 & 6 & -3.592173000 & 1.632204000 & -0.000009000 \\
\hline 7 & -1.517790000 & -2.053328000 & 0.003239000 & 7 & -2.215294000 & 1.619346000 & -0.000021000 \\
\hline 6 & -3.922963000 & -1.566914000 & 0.016151000 & 6 & -4.395055000 & 0.507285000 & -0.000014000 \\
\hline 6 & -3.877914000 & -0.188751000 & 0.000487000 & 6 & -3.975359000 & -0.825728000 & -0.000024000 \\
\hline 6 & -5.078661000 & 0.627655000 & -0.007415000 & 6 & -4.921943000 & -1.923831000 & -0.000056000 \\
\hline 6 & -4.655550000 & 1.908420000 & -0.021114000 & 6 & -4.177966000 & -3.051849000 & -0.000014000 \\
\hline 6 & -3.196335000 & 1.855949000 & -0.019698000 & 6 & -2.788200000 & -2.621486000 & -0.000031000 \\
\hline 7 & -2.744021000 & 0.594920000 & -0.008179000 & 7 & -2.687815000 & -1.270665000 & -0.000040000 \\
\hline 6 & -2.470063000 & 3.068702000 & -0.024298000 & 6 & -1.774949000 & -3.589647000 & -0.000022000 \\
\hline 6 & -1.132579000 & 3.383497000 & -0.012893000 & 6 & -0.392061000 & -3.551614000 & -0.000014000 \\
\hline 6 & -0.674924000 & 4.752548000 & -0.008484000 & 6 & 0.406338000 & -4.738305000 & -0.000001000 \\
\hline 6 & 0.674923000 & 4.752548000 & 0.008483000 & 6 & 1.717142000 & -4.373040000 & -0.000004000 \\
\hline 6 & 1.132579000 & 3.383497000 & 0.012892000 & 6 & 1.779934000 & -2.944923000 & -0.000001000 \\
\hline 7 & 0.000000000 & 2.595181000 & 0.000000000 & 7 & 0.488246000 & -2.487207000 & -0.000011000 \\
\hline 6 & 2.470063000 & 3.068703000 & 0.024297000 & 6 & 2.987956000 & -2.248554000 & 0.000001000 \\
\hline 6 & 3.196335000 & 1.855949000 & 0.019698000 & 6 & 3.291222000 & -0.902083000 & -0.000002000 \\
\hline 6 & 4.655550000 & 1.908420000 & 0.021116000 & 6 & 4.726781000 & -0.509095000 & -0.000004000 \\
\hline 6 & 5.078660000 & 0.627655000 & 0.007415000 & 8 & 4.713402000 & 0.853304000 & 0.000067000 \\
\hline 6 & 3.877914000 & -0.188751000 & -0.000486000 & 6 & 3.387708000 & 1.240895000 & -0.000005000 \\
\hline 6 & 3.922963000 & -1.566914000 & -0.016151000 & 6 & 3.134472000 & 2.590377000 & 0.000013000 \\
\hline 7 & 2.744021000 & 0.594920000 & 0.008180000 & 7 & 2.531720000 & 0.208104000 & 0.000011000 \\
\hline 1 & 1.272639000 & -1.067596000 & 0.018702000 & 1 & 0.696754000 & 1.420061000 & -0.000062000 \\
\hline 1 & 0.000000000 & 1.586033000 & 0.000000000 & 1 & 0.211548000 & -1.516467000 & -0.000015000 \\
\hline 1 & -1.272639000 & -1.067596000 & -0.018702000 & 1 & -1.708617000 & 0.739286000 & -0.000039000 \\
\hline 1 & 3.743124000 & -4.459543000 & -0.057311000 & 1 & 2.200270000 & 5.338700000 & 0.000048000 \\
\hline 1 & 1.188396000 & -5.310614000 & -0.045232000 & 1 & -0.483831000 & 5.494916000 & 0.000014000 \\
\hline 1 & -1.188395000 & -5.310614000 & 0.045232000 & 1 & -2.800277000 & 4.846683000 & 0.000022000 \\
\hline 1 & -3.743124000 & -4.459543000 & 0.057310000 & 1 & -5.017854000 & 3.332850000 & 0.000019000 \\
\hline 1 & -6.089048000 & 0.245206000 & -0.002912000 & 1 & -5.996978000 & -1.818089000 & -0.000072000 \\
\hline 1 & -5.242576000 & 2.815406000 & -0.029915000 & 1 & -4.507570000 & -4.080753000 & 0.000001000 \\
\hline 1 & -1.342916000 & 5.600190000 & -0.016380000 & 1 & -0.006738000 & -5.735375000 & 0.000002000 \\
\hline 1 & 1.342915000 & 5.600190000 & 0.016379000 & 1 & 2.588584000 & -5.009167000 & -0.000001000 \\
\hline 1 & 5.242575000 & 2.815407000 & 0.029918000 & 1 & -5.464126000 & 0.685945000 & -0.000009000 \\
\hline 1 & 6.089048000 & 0.245206000 & 0.002911000 & 1 & -2.152021000 & -4.607183000 & -0.000016000 \\
\hline 1 & -4.902960000 & -2.030583000 & 0.025465000 & 1 & 3.863757000 & -2.888234000 & -0.000002000 \\
\hline 1 & -3.101770000 & 3.950279000 & -0.033650000 & 1 & 3.983730000 & 3.259037000 & 0.000041000 \\
\hline 1 & 3.101770000 & 3.950279000 & 0.033650000 & 8 & 5.730558000 & -1.159315000 & 0.000116000 \\
\hline 1 & 4.902960000 & -2.030583000 & -0.025465000 & & & & \\
\hline \multicolumn{4}{|c|}{ M2 } & \multicolumn{4}{|c|}{ C1 } \\
\hline 6 & 2.401477000 & -4.033050000 & 0.000126000 & 6 & -2.838214000 & 4.052836000 & -0.002647000 \\
\hline 6 & 1.065552000 & -4.410359000 & 0.000059000 & 6 & -1.551166000 & 4.491331000 & 0.002740000 \\
\hline 6 & 0.293400000 & -3.237545000 & 0.000042000 & 6 & -0.689965000 & 3.343379000 & 0.014047000 \\
\hline 6 & 2.447783000 & -2.631078000 & 0.000028000 & 6 & -2.819800000 & 2.615425000 & 0.004378000 \\
\hline 7 & 1.153336000 & -2.178471000 & 0.000167000 & 7 & -1.504837000 & 2.236493000 & 0.016716000 \\
\hline 6 & -1.126755000 & -3.145453000 & -0.000005000 & 6 & 0.689968000 & 3.343378000 & 0.014066000 \\
\hline 6 & -2.034863000 & -4.225166000 & -0.000067000 & 6 & 1.551169000 & 4.491330000 & 0.002798000 \\
\hline 6 & -3.310958000 & -3.696376000 & -0.000105000 & 6 & 2.838218000 & 4.052835000 & -0.002542000 \\
\hline 6 & -3.194879000 & -2.291840000 & -0.000060000 & 6 & 2.819802000 & 2.615423000 & 0.004445000 \\
\hline
\end{tabular}




\begin{tabular}{|c|c|c|c|c|c|c|c|}
\hline 7 & -1.851073000 & -1.995605000 & 0.000050000 & 7 & 1.504838000 & 2.236491000 & 0.016738000 \\
\hline 6 & -4.215678000 & -1.337652000 & -0.000090000 & 6 & 3.895694000 & 1.753758000 & -0.003607000 \\
\hline 6 & -4.087222000 & 0.036538000 & -0.000015000 & 6 & 3.770746000 & 0.361299000 & -0.006272000 \\
\hline 6 & -5.234068000 & 0.920438000 & 0.000286000 & 8 & 4.950444000 & -0.346768000 & -0.011402000 \\
\hline 6 & -4.734021000 & 2.175929000 & -0.000201000 & 6 & 4.611761000 & -1.675195000 & -0.011713000 \\
\hline 6 & -3.285124000 & 2.034216000 & 0.000107000 & 6 & 3.130774000 & -1.682139000 & -0.007258000 \\
\hline 7 & -2.906243000 & 0.752370000 & 0.000167000 & 7 & 2.684394000 & -0.386321000 & -0.004476000 \\
\hline 6 & -2.482041000 & 3.209414000 & 0.000058000 & 6 & 2.483667000 & -2.883755000 & -0.002471000 \\
\hline 6 & -1.138407000 & 3.452526000 & 0.000075000 & 6 & 1.123278000 & -3.251564000 & 0.003866000 \\
\hline 6 & -0.602027000 & 4.796293000 & 0.000035000 & 6 & 0.688467000 & -4.593805000 & 0.031557000 \\
\hline 6 & 0.743540000 & 4.723803000 & 0.000071000 & 6 & -0.688469000 & -4.593805000 & 0.031579000 \\
\hline 6 & 1.122179000 & 3.326287000 & 0.000066000 & 6 & -1.123280000 & -3.251563000 & 0.003898000 \\
\hline 7 & -0.042588000 & 2.601727000 & 0.000115000 & 7 & -0.000001000 & -2.460709000 & -0.011437000 \\
\hline 6 & 2.437591000 & 2.930348000 & 0.000054000 & 6 & -2.483670000 & -2.883755000 & -0.002399000 \\
\hline 6 & 3.005456000 & 1.645796000 & 0.000021000 & 6 & -3.130776000 & -1.682138000 & -0.007160000 \\
\hline 8 & 4.388385000 & 1.664475000 & -0.000163000 & 6 & -4.611763000 & -1.675193000 & -0.011507000 \\
\hline 6 & 4.804468000 & 0.359184000 & -0.000274000 & 8 & -4.950444000 & -0.346765000 & -0.011454000 \\
\hline 6 & 3.555815000 & -0.422079000 & -0.000088000 & 6 & -3.770746000 & 0.361301000 & -0.006267000 \\
\hline 6 & 3.574582000 & -1.781691000 & -0.000047000 & 6 & -3.895692000 & 1.753761000 & -0.003686000 \\
\hline 7 & 2.480220000 & 0.447795000 & -0.000142000 & 7 & -2.684394000 & -0.386320000 & -0.004373000 \\
\hline 1 & 0.932719000 & -1.189806000 & 0.000233000 & 1 & -1.243864000 & 1.256831000 & 0.026925000 \\
\hline 1 & -0.096074000 & 1.593919000 & 0.000146000 & 1 & -0.000002000 & -1.451511000 & -0.053207000 \\
\hline 1 & -1.535003000 & -1.030292000 & 0.000129000 & 1 & 1.243864000 & 1.256830000 & 0.026919000 \\
\hline 1 & 3.267244000 & -4.676585000 & 0.000154000 & 1 & -3.738832000 & 4.646456000 & -0.012905000 \\
\hline 1 & 0.670608000 & -5.413657000 & 0.000040000 & 1 & -1.209587000 & 5.514283000 & -0.002213000 \\
\hline 1 & -1.755770000 & -5.266379000 & -0.000093000 & 1 & 1.209591000 & 5.514283000 & -0.002148000 \\
\hline 1 & -4.246671000 & -4.233878000 & -0.000168000 & 1 & 3.738836000 & 4.646454000 & -0.012756000 \\
\hline 1 & -6.265529000 & 0.599407000 & 0.000431000 & 8 & 5.421677000 & -2.554355000 & -0.014922000 \\
\hline 1 & -5.265791000 & 3.116261000 & -0.000418000 & 1 & 1.353288000 & -5.443242000 & 0.051801000 \\
\hline 1 & -1.222240000 & 5.679766000 & 0.000000000 & 1 & -1.353289000 & -5.443241000 & 0.051845000 \\
\hline 1 & 1.459397000 & 5.531290000 & 0.000063000 & 8 & -5.421679000 & -2.554352000 & -0.014976000 \\
\hline 1 & -5.225133000 & -1.734150000 & -0.000125000 & 1 & 4.894178000 & 2.166373000 & -0.010288000 \\
\hline 1 & -3.066254000 & 4.123502000 & -0.000044000 & 1 & 3.158699000 & -3.734137000 & 0.000576000 \\
\hline 1 & 3.161470000 & 3.733770000 & 0.000046000 & 1 & -3.158702000 & -3.734136000 & 0.000675000 \\
\hline 1 & 4.550926000 & -2.252671000 & -0.000093000 & 1 & -4.894176000 & 2.166376000 & -0.010430000 \\
\hline 8 & 5.957332000 & 0.039761000 & -0.000244000 & & & & \\
\hline \multicolumn{4}{|c|}{$\mathrm{C} 2$} & \multicolumn{4}{|c|}{$\mathbf{T}$} \\
\hline 6 & 2.891885000 & -3.899560000 & -0.069401000 & 6 & 3.351302000 & 3.557259000 & 0.000137000 \\
\hline 6 & 1.584967000 & -4.371825000 & -0.066164000 & 6 & 2.124261000 & 4.193823000 & 0.000215000 \\
\hline 6 & 0.732926000 & -3.258172000 & -0.021153000 & 6 & 1.126129000 & 3.197567000 & 0.000076000 \\
\hline 6 & 2.838703000 & -2.499949000 & -0.027563000 & 6 & 3.115967000 & 2.168491000 & -0.000013000 \\
\hline 7 & 1.512355000 & -2.141765000 & -0.000782000 & 7 & 1.754809000 & 1.987557000 & -0.000054000 \\
\hline 6 & -0.695870000 & -3.262665000 & 0.001419000 & 6 & -0.277138000 & 3.391517000 & 0.000091000 \\
\hline 6 & -1.539742000 & -4.381639000 & 0.060297000 & 6 & -0.972459000 & 4.616837000 & 0.000271000 \\
\hline 6 & -2.849702000 & -3.918211000 & 0.070470000 & 6 & -2.325590000 & 4.330844000 & 0.000251000 \\
\hline 6 & -2.806729000 & -2.518623000 & 0.019784000 & 6 & -2.469948000 & 2.928583000 & 0.000066000 \\
\hline 7 & -1.483033000 & -2.151281000 & -0.020873000 & 7 & -1.208386000 & 2.392823000 & -0.000048000 \\
\hline 6 & -3.875745000 & -1.599969000 & 0.016758000 & 6 & -3.655774000 & 2.177952000 & 0.000030000 \\
\hline 6 & -3.785622000 & -0.243185000 & -0.001852000 & 6 & -3.733015000 & 0.812372000 & -0.000196000 \\
\hline 6 & -4.986523000 & 0.603218000 & -0.000892000 & 8 & -4.991528000 & 0.245875000 & -0.000010000 \\
\hline 8 & -4.494049000 & 1.887310000 & -0.015164000 & 6 & -4.818575000 & -1.103904000 & -0.000682000 \\
\hline 6 & -3.119842000 & 1.787525000 & -0.021890000 & 6 & -3.343768000 & -1.294330000 & -0.000319000 \\
\hline 7 & -2.659387000 & 0.570105000 & -0.016905000 & 7 & -2.743777000 & -0.105808000 & -0.000446000 \\
\hline 6 & -2.476236000 & 3.049042000 & -0.030911000 & 6 & -2.851738000 & -2.603449000 & -0.000112000 \\
\hline 6 & -1.151949000 & 3.372640000 & -0.019317000 & 6 & -1.578204000 & -3.122850000 & 0.000034000 \\
\hline 6 & -0.694258000 & 4.751147000 & -0.028625000 & 6 & -1.319218000 & -4.540786000 & 0.000295000 \\
\hline 6 & 0.649209000 & 4.752625000 & -0.007819000 & 6 & 0.018817000 & -4.729909000 & 0.000418000 \\
\hline 6 & 1.110693000 & 3.375151000 & 0.013025000 & 6 & 0.658634000 & -3.436238000 & 0.000183000 \\
\hline 7 & -0.019891000 & 2.584196000 & 0.004628000 & 7 & -0.348631000 & -2.498686000 & -0.000031000 \\
\hline 6 & 2.437137000 & 3.060015000 & 0.030583000 & 6 & 2.024581000 & -3.291406000 & 0.000203000 \\
\hline 6 & 3.096112000 & 1.806203000 & 0.030801000 & 6 & 2.830865000 & -2.142344000 & -0.000080000 \\
\hline
\end{tabular}




\begin{tabular}{|rrrr|rrrr|}
\hline 8 & 4.469089000 & 1.924912000 & 0.032908000 & 8 & 4.182660000 & -2.423901000 & 0.000209000 \\
6 & 4.979625000 & 0.647888000 & 0.019750000 & 6 & 4.841053000 & -1.221324000 & -0.000633000 \\
6 & 3.790840000 & -0.215118000 & 0.010844000 & 6 & 3.764609000 & -0.218309000 & -0.000314000 \\
6 & 3.898428000 & -1.570692000 & -0.013482000 & 6 & 4.046222000 & 1.119235000 & -0.000118000 \\
7 & 2.653189000 & 0.582496000 & 0.021362000 & 7 & 2.546883000 & -0.862715000 & -0.000446000 \\
1 & 1.225940000 & -1.171754000 & 0.055850000 & 1 & 1.342564000 & 1.062171000 & -0.000159000 \\
1 & -0.022007000 & 1.574505000 & 0.029058000 & 1 & -0.203756000 & -1.499580000 & -0.000211000 \\
1 & -1.201967000 & -1.180701000 & -0.092886000 & 1 & -1.055363000 & 1.392084000 & -0.000181000 \\
1 & 3.800397000 & -4.480395000 & -0.102254000 & 1 & 4.329353000 & 4.012437000 & 0.000195000 \\
1 & 1.260960000 & -5.399557000 & -0.098907000 & 1 & 1.937583000 & 5.255726000 & 0.000346000 \\
1 & -1.208331000 & -5.406779000 & 0.099652000 & 1 & -0.510231000 & 5.590892000 & 0.000413000 \\
1 & -3.754053000 & -4.504628000 & 0.116263000 & 1 & -3.146160000 & 5.031184000 & 0.000374000 \\
1 & -1.364931000 & 5.596187000 & -0.049551000 & 1 & -2.100084000 & -5.285594000 & 0.000393000 \\
1 & 1.318295000 & 5.599182000 & -0.009043000 & 1 & 0.563364000 & -5.661475000 & 0.000625000 \\
1 & -4.878720000 & -2.012409000 & 0.035288000 & 1 & -4.590613000 & 2.722476000 & 0.000214000 \\
1 & -3.158556000 & 3.888509000 & -0.044435000 & 1 & -3.636858000 & -3.351758000 & -0.000078000 \\
1 & 3.111761000 & 3.905863000 & 0.035242000 & 1 & 2.582970000 & -4.218266000 & 0.000469000 \\
1 & 4.906188000 & -1.971396000 & -0.025321000 & 1 & 5.096267000 & 1.388966000 & -0.000101000 \\
8 & 6.152717000 & 0.415808000 & 0.016520000 & 8 & 6.033979000 & -1.127292000 & 0.000351000 \\
8 & -6.156189000 & 0.354724000 & 0.009619000 & 8 & -5.719785000 & -1.890838000 & 0.000207000 \\
\hline
\end{tabular}

